\title{
A REGISTRATION METHOD OF POINT CLOUDS COLLECTED BY MOBILE LIDAR USING SOLELY STANDARD LAS FILES INFORMATION
}

\author{
L. Gézero ${ }^{\mathrm{a}, \mathrm{b}}, \mathrm{C}$. Antunes ${ }^{\mathrm{c}}$ \\ ${ }^{a}$ Faculdade de Ciências, Universidade de Lisboa, Campo Grande 1749-016 Lisbon Portugal \\ ${ }^{\text {b }}$ LandCOBA, Consultores de Sistemas de Informação e Cartografia Digital Lda - 1.gezero@ cobagroup.com \\ c Instituto Dom Luiz, Faculdade de Ciências, Universidade de Lisboa, Campo Grande 1749-016 Lisbon, Portugal - \\ cmantunes@fc.ul.pt
}

KEY WORDS: Mobile systems, point cloud, LiDAR, registration, LAS, trajectory adjustment

\begin{abstract}
:
In the last few years, LiDAR sensors installed in terrestrial vehicles have been revealed as an efficient method to collect very dense 3D georeferenced information. The possibility of creating very dense point clouds representing the surface surrounding the sensor, at a given moment, in a very fast, detailed and easy way, shows the potential of this technology to be used for cartography and digital terrain models production in large scale. However, there are still some limitations associated with the use of this technology. When several acquisitions of the same area with the same device, are made, differences between the clouds can be observed. The range of that differences can go from few centimetres to some several tens of centimetres, mainly in urban and high vegetation areas where the occultation of the GNSS system introduces a degradation of the georeferenced trajectory. Along this article a different method point cloud registration is proposed. In addition to the efficiency and speed of execution, the main advantages of the method are related to the fact that the adjustment is continuously made over the trajectory, based on the GPS time. The process is fully automatic and only information recorded in the standard LAS files is used, without the need for any auxiliary information, in particular regarding the trajectory.
\end{abstract}

\section{INTRODUCTION}

Mobile LiDAR Systems (MLS) collect information that allows the generation of very dense and detailed 3D point clouds. The high density and precision of these clouds combined with the high speed of information gathering have attracted the interest of producers and users of georeferenced geographic information at large scales. Cost reduction, high detail and ease acquisition make this technology one of the most promising forms of geographic information acquisition that has emerged in recent years. However, there are still some limitations associated with the processing and information extraction, which limits the wider use of this type of technology. One of these limitations results from the fact that, when different clouds are acquired over the same geographic areas, at different times and / or from different points of view, there are differences in the alignment between these clouds (Gao et al., 2015; Gressin et al. 2012). These differences can result from factors inherent to the system itself, such as the calibration process, drifts and instrument accuracy. However, the factor that is proven to be of greater influence and more difficult to control results from the obstructions to the GNSS signal, in particular in urban areas or with dense vegetation, causing a degradation in the quality of the GNSS solution and consequently a greater error in the position of vehicle along its trajectory (Shaer and Vallet, 2016).

The efficient registration of point clouds has thus been a challenge for many researchers in recent years (Gonzalez et al., 2015; Pomerlau et al., 2015; Wang and Ferrie, 2015). Often the problem results more from the relative inconsistency between clouds than from the deterioration of the absolute point coordinates of the cloud, since these differences are usually small. Cloud inconsistency prevents the execution of algorithms of automatic object extraction, automatic generation of digital terrain models, etc.. Throughout this work, a method of point cloud registration collected from MLS is proposed. In addition to the speed of execution, the main advantages of this method are related to the fact that the adjustment is made from the reconstruction of the vehicle's trajectory. This allows to apply the corrections continuously over time and rewrite the adjusted cloud in the same way as it was acquired. This represents a great advantage over iterative or non-iterative methods, which require a pre-initialization of the clouds or identification of objects on the clouds. Given the density of the information it will always be necessarily slower. Another equally important advantage is that for the application of the method only information recorded in the standard LAS files is used, without the need for any auxiliary information, in particular what regards to the trajectory. Given the increasing amount of MLS's and software of data processing and visualization, standardization is important to allow a greater and easier information sharing. Through the application of this method we demonstrate that it is possible to adjust the clouds only with the information contained in the standard files, rebuilding the trajectory of the vehicle automatically, without using any other auxiliary information. This is also useful for those that nowadays are increasingly specialized in the automatic extraction of information from clouds. Using just the LAS information there is no need to know the collection process and the system used to make the registration. We believe that the dissemination of this type of methods is a contribution to encourage the use of standardized information.

Finally, it is important to mention that the application of the method is fully automatic. This implementation is designed to perform the vertical registration of the clouds (since this is the largest error associated with the GNSS system), however, the method can be easily transposed to perform the horizontal registration and even to compensate transverse inclination between clouds, which results from the boresight or drift errors of the Inertial Measurement Unit (IMU), as it happened in the practical case that is presented.

The paper is organized as follows. Section 2 presents a brief explanation of the MLS working principles and a description of the main point cloud file formats, including the LAS standard file format. Section 2 ends with the description of the three main steps of the presented method. In Section 3, a practical case study of 
the method application is portrayed. In this case, two different registration methods are applied. One for the absolute registration of the anchor clouds block, then the present method is applied to make the registration of block B clouds to block A. Conclusions and future work are provided in Section 4.

\section{DEFINITIONS AND METHOD DESCRIPTION}

\subsection{MLS working principles}

Since the proposed method is based on the principles of data collection, it is important to introduce them.

The travelled trajectory of the vehicle carrying the LiDAR sensor along time can be represented by a succession of points. The coordinates of these points are determined by the georeferencing system, which is an inertial system based on the "closed loop" between the information provided by the GNSS antenna, at time intervals (typically $1 \mathrm{Hertz}$ ) and the intermediate positions calculated by the IMU according to the registration frequency (typically 0.05 Hertz). Auxiliary sensors, such as a second GNSS antenna and a precision odometer, are often used to improve the solution obtained for each position (El Sheimy, 2005). In areas with obstructions to the GNSS signal, particularly in urban areas and / or with high vegetation, the accuracy of the coordinates provided by the GNSS system are severely degraded. In these areas, the IMU allows the maintenance of the precision of the coordinates for periods of time relatively long according to the IMU quality. Depending on the type of the IMU used in commercial MLS, interruptions greater than 30-60 seconds cause significant errors in the trajectory which, according to the extension of the degradation period, may range from a few centimetres to 1- 2 meters. Such errors are not satisfactory for most applications of point clouds (Shaer and Vallet, 2016). The final result of the trajectory is usually obtained through the application of a Kalman filter, which allows the optimization of the position estimation by compensating the GNSS / IMU errors (Abuhadrous et al., 2003). There are several solutions to correct these errors that are based on more or less complicated mathematical models depending on the quality of the IMU (El Sheimy, 2005). At each position of the trajectory it is possible to associate a set of values related to the moment and conditions of acquisition, namely GPS time, platform speed, registration angle, etc.. In order to obtain the georeferenced point cloud, it is necessary to integrate the information from each position that describe the trajectory with the information collected by the LiDAR sensor.

The principle of the LiDAR sensor is based on the emission of LASER pulses, which after reflection on a certain object are again recorded in the sensor. Essentially, there are three methods for determining the distance between the reflected point on the object and the sensor: triangulation; measurement of the wave phase difference; and measurement of the travelling time (Puente et al., 2013).

In order to obtain as much information as possible about the surface surrounding the sensor at a given moment, two types of movement are performed by the sensor. The first, is the rotational movement around sensor axis, this allows gathering the information concerning a profile and is usually made through a rotating mirror that deflects the direction of the pulse emitted by the laser. The second movement associated to the sensor results from the movement of the vehicle itself, which allows changing the position of the sensor and obtaining a cloud of points representing the surrounding reality (Figure 1).

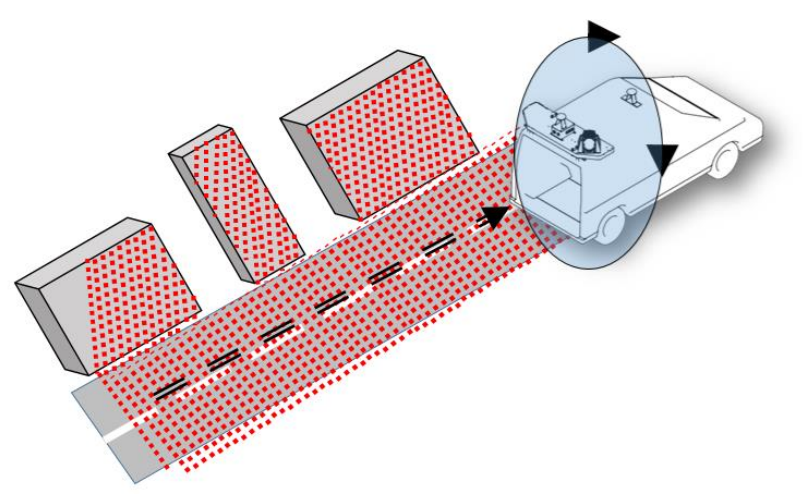

Figure 1. Principal movement components of Mobile LiDAR System.

There are two frequency values associated with the LIDAR sensor. The sensor scan frequency, which represents the number of pulses emitted by the sensor in a period of time, and the time interval between each recorded position of the trajectory, which is determined by the recording frequency of the IMU. While the spatial interval between the positions depends on the speed of the vehicle, the transverse density of the point cloud depends only on the scan frequency of the sensor, and the longitudinal density is directly related to the mirror rotation frequency and the vehicle speed. Each of the positions of the trajectory is integrated with the angles and distances measured by the sensor along a complete rotation of the mirror, in order to obtain a set of georeferenced points. The repetition of this process for all positions along the trajectory, allows to obtain the cloud of georeferenced points. It is possible to associate to each point of the final cloud the GPS time of the position of the trajectory in which they were obtained, being that GPS time equal for all points collected during the same rotation of the mirror. On the other hand, it is also possible to associate to each point of the cloud the information concerning the scan angle. This and other information regarding the acquisition process can be stored as attributes of each point.

\subsection{Point clouds file formats}

There is a large and increasing number of file formats that allow the storage of point clouds. The point clouds file format can be classified according to its coding form in two types, the ASCII (American Standard Coding for Information Interchange), commonly referred as text files, and the binary files. The simplest ASCII $x y z$ file format contains only the information concerning the position of the points, namely their 3-dimensional coordinates. Other examples of ASCII files are the pts files which, in addition to the coordinates, allow the storage of the intensity value and the three RGB values, and the $p t x$ files (Leica Geosystems) that allow the storage of several point clouds in a single file.

The binary file formats have a fairly large diversity, both in the way the information is encoded and in the type of coded information. This diversity of formats results from the commercial strategies of system and software manufacturers to improve processing, visualization, disk space optimization and access speed. Most of these binary formats are copyrighted, so their structure and format are unknown. That fact prevents the interoperability between systems, as well as the use of the information in open source software. For additional information about the file formats, please refer to Samberg (2007). 
The use of ASCII formats for interoperability between systems is limited for performance and readability issues. The extra step of parsing the text to binary, in order to allow its interpretation by the software, necessarily makes the process of reading the information slower. Apart from that, the disk space occupied by an ASCII file is substantially higher than for a binary file, containing the same information.

The American Society for Photogrammetry \& Remote Sensing (ASPRS) through its Committee LASer (LAS) File Format Exchange Activities created the LAS file format. The LAS is a public format that allows the interoperability of threedimensional point clouds and whose structure allows to store the specific information associated to the LiDAR data collection process, without being too complex. The goal of LAS is to provide an open format, containing the information regarding a cloud of points generated by a LiDAR system (or any other source). It should be an option to export the different commercial software and maintain information related to the GPS system, IMU and laser sensor that originated each of the registered points (X, Y, Z) (ASPRS, 2013).

At the date of this document, the most recent version of the LAS format is the 1.4 whose structure is divided into 4 main blocks of information: a header, Variable Length Records, individual records of each of the points and finally the Extended Variable Length Records. All information is binary encoded in littleendian (ASPRS, 2013). The header contains generic information associated with the file, such as number of points, geographical boundaries, etc.. For the version 1.4, the individual records of each point have the formats $0-10$. These formats result from the evolution of the LAS versions based on the detected needs, allowing to increase the information associated to each point or increase the number of bytes available for its registration. For each point in addition to their coordinates, the format allows to store specific information, namely GPS time, RGB values, intensity value, number of returns, registration angle, etc.. Throughout this work we intend to take advantage of the information associated with each point, to carry out the registration process between clouds, namely the GPS time and the scan angle.

\subsection{Method description}

As referred in Section 2.1, the largest error associated to the trajectory of a MLS is the altimetry component. That results from the fact that the main sensor of the MLS georeferencing system is the GNSS sensor. Although all the methodology presented here focuses specifically on registration in altimetry, the whole process can be easily adapted to the planimetry adjustment between the clouds.

Along this process description, two clouds are considered with an overlapping area. One cloud has fixed coordinates (anchor cloud) and another cloud has variable vertical differences related to the first one. The purpose of the method is to eliminate the differences between the two clouds by adjusting the second cloud to fit the anchor cloud.

The method is divided in three main steps: 1) Definition of the points for adjustment along the trajectory; 2) Determination of the corrections to be applied; 3) Rewriting a new cloud adjusted, based on the corrected trajectory. All steps are performed automatically, based on previously defined threshold values.

Step 1 - As mentioned in Section 2.1, the trajectory of the vehicle carrying the sensor can be represented by a sequence of points showing the position of the Laser sensor over time. In this step we intend to reconstruct the vertical projection of the trajectory points in the ground. To obtain these points it is necessary to know the scan angle $(\alpha)$ of each point. Since the origin of the scan angle coordinate system is vertical down direction, all points that have $\alpha=0$ are under the sensor nadir (Figure 2a). If we visualize a cloud by associating to each fixed angular range a different color, we can see that the areas covered by each angular range increase symmetrically as they move away from the vehicle trajectory (Figure $2 b$ ).
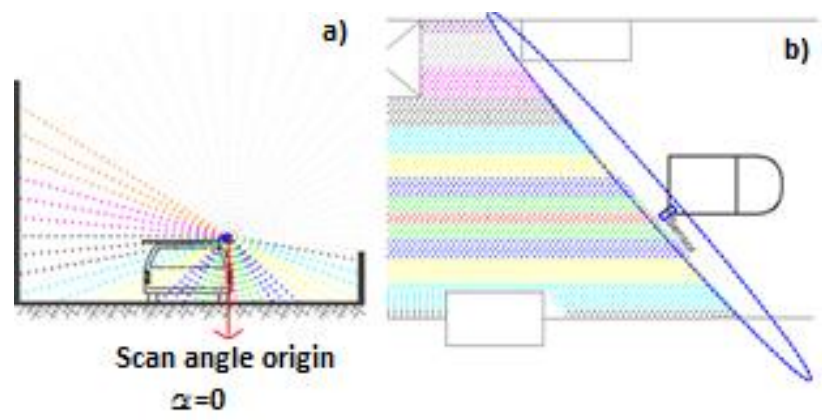

Figure 2. a) Scan angle origin. b) Coloured angular range.

To reconstruct the trajectory of the vehicle, besides the scan angle, it is necessary to know the GPS time of each point of the cloud, in the way to establish the time interval for reconstruction of the trajectory points. It should be noted that the GPS time of each point corresponds to the moment of registration of the IMU, so all points obtained along a rotation of the laser mirror have the same GPS time. Figure 3 shows in red the points resulting from a pre-established GPS time interval $(\tau)$.

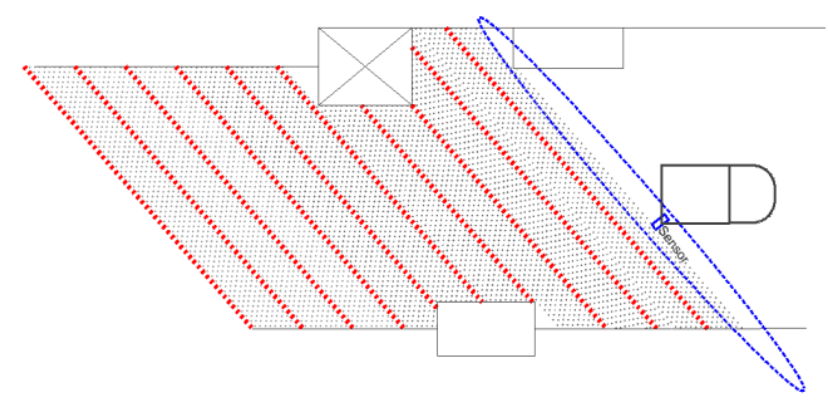

Figure 3. GPS time interval.

From the intersection of the two variables $(\alpha, \tau)$, it is possible to establish a set of points with a time interval $t$ representing the projection of the trajectory carried out by MLS on the points cloud (Figure 4).

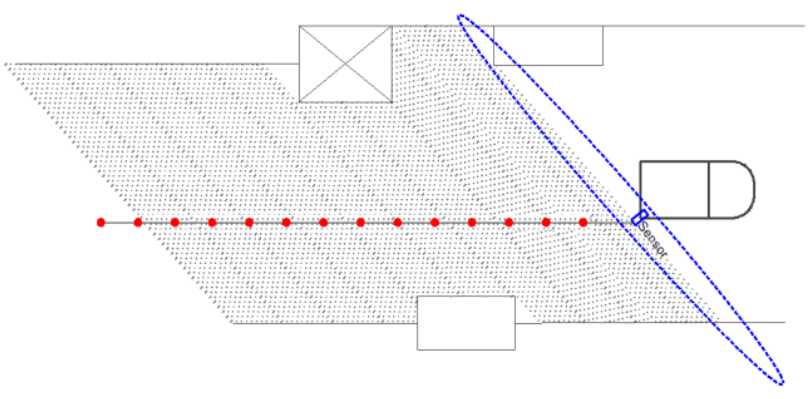

Figure 4. Trajectory points of the MLS.

The values of the scan angle and GPS time of each point can be directly read from the LAS file. In LAS file version 1.1 to 1.3 , 
the scan angle field is limited to 1 byte. The maximum value recorded is then $255 \mathrm{so}$, to store the $360^{\circ}$, the maximum interval resolution stored is $360 / 255$. In version 1.4 , for a point format greater than 6 , the scan angle field has been extended to two bytes to support finer angle resolution. The scan angle field is a signed short type, which represents the rotational position of the emitted Laser pulse with respect to the vertical of the coordinate system of the data. Down in the data coordinate system is the 0.0 position (Figure 2a). Each increment represents 0.006 degrees.

The GPS time was included, as 8 bytes double, in all LAS versions point format 1-10. The value is GPS Week Time if the Global Encoding low bit is clear and Adjusted Standard GPS Time if the Global Encoding low bit is set. The Global Encoding is available in the Public Header Block and it is common to all points in file. Since for the time interval $\tau$ the Euclidean distance between consecutive points depend on the velocity of the vehicle, a third restriction of minimum distance between consecutive points $(\Delta)$ should be applied. That restriction avoids very tied points when the velocity is low or very low.

The final result of the trajectory points can be represented as the intersection of the three previous restrictions (Equation 1).

$$
T i=\left\{\begin{array}{c}
P(\tau)_{i}, P(\tau+1)_{i+1}, P(\tau+2)_{i+2}, \ldots, P(\tau+N)_{i+N} \\
P(\alpha)_{i}, P(\alpha)_{i+1}, P(\alpha)_{i+2}, \ldots, P(\alpha)_{i+N}, \alpha=0 \\
\left|P_{i-} P_{i-1}\right| \geq \Delta
\end{array}\right.
$$

where $T_{i}=$ final trajectory

$\mathrm{P}_{\mathrm{i}}=$ all available trajectory points

$\tau=$ chosen GPS time interval

$\alpha=$ scan angle ( $\alpha=0$ over the trajectory)

$\Delta=$ minimum distance between consecutive points

Step 2 - After the identification of the points along the trajectory it is necessary to measure the distance between the two clouds in each of these points. To do so, a circle of radius $r$ is centred in each of the points resulting from Step 1 . Those circles are used to identify all points from both clouds in the neighbour of the trajectory points. Since the cloud area along the trajectory used to establish the comparison between the clouds is in the middle of the road and immediately under the sensor, it is unlikely that any object can cause a cloud deformation, so it is likely that the points identified inside the circle create approximately a plane. However, in order to eliminate the noise caused by some undergrowth or any other small object under the vehicle at the time of capture, the RANdom Sample Consensus (RANSAC) method can be used to ensure the establishment of the plane in the two clouds and determine the vertical differences between them. It is assumed, however, that the planes formed in the two clouds are parallel. Initially proposed by Fischler and Bolles (1981), the RANSAC method consists of estimating a parameter to cope with the existence of a large proportion of outliers. RANSAC is a sampling technique that generates candidate solutions using a set of observations (in this case points) necessary to estimate the parameters underlying the model. Unlike more conventional sampling techniques that use as much information as possible to obtain an initial solution and advance to eliminate the outliers, the RANSAC algorithm starts the process with minimum possible data set and will increase this set with new data consistent with the model.

Using the RANSAC method with the plane equation it is possible to define planes in both clouds and the difference between those two planes correspond to the average difference between the two clouds in each trajectory point (Figure 5).

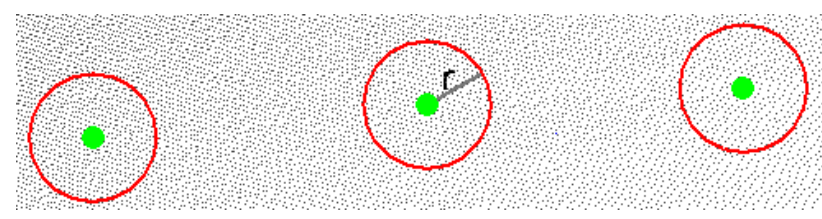

Figure 5. Circle (with radius $r$ ) in each trajectory point.

Then next task is to interpolate the difference values, in order to associate these values to all the GPS epochs existing in the point cloud (IMU registration values). This interpolation is possible due to the fact that the trajectories are obtained through the application of a Kalman filter, which allows to optimize the estimation of the positions (Gressin et al., 2012).

Since the distance between the points used to measure the vertical differences between the clouds is small and the resulting trajectory is smooth due to the Kalman filter, a linear interpolator is expected to be sufficient. However, a quadratic or higher-grade interpolator can also be used to perform that interpolation. It is noteworthy that the interpolation is performed based on the time difference between the points, instead of the spatial difference. Considering a limited time interval for two of the trajectory points where the differences between the clouds are known $\left(\mathrm{v}_{\mathrm{i}}\right.$, $\left.\mathrm{V}_{\mathrm{i}+1}\right)$, as well as its corresponding GPS epochs $\left(\mathrm{E}_{\mathrm{i}}, \mathrm{E}_{\mathrm{i}+1}\right)$, any GPS intermediate instant $\left(\mathrm{E}_{\mathrm{j}}\right)$ can be interpolated using Equation 2. It is possible to interpolate the difference values for all GPS epochs in the cloud, using the LAS file information for each point of the cloud.

$$
E_{j}=v_{i}+\frac{\left(v_{i+1}-v_{i-1}\right)}{\left(E_{i+1}-E_{i}\right)}\left(E_{j}-E_{i}\right), \quad E_{i}<E j<E_{i+1}
$$

where $\quad E_{j}=$ GPS epoch to be interpolated

$\mathrm{E}_{\mathrm{i}}=$ GPS epoch of the initial interval trajectory point $\mathrm{E}_{\mathrm{i}+1}=$ GPS epoch of the final interval trajectory point $\mathrm{v}_{\mathrm{i}}=$ vertical difference at initial interval trajectory point $\mathrm{v}_{\mathrm{i}+1}=$ vertical difference at final interval trajectory

point

Step 3 - At the end of Step 2, all points in the cloud should have an associated value of the difference. Finally, a new point cloud can be generated, applying the correction based in the values of cloud difference in each point. The new point cloud will be adjusted to the anchor cloud in all the GPS epochs, eliminating the vertical differences along the original trajectory.

Instead of using just vertical differences, horizontal differences also can be equally used. Those values can be obtained from a similar process to Step 2, looking for vertical planes or even through field measured control points.

\section{RESULTS}

\subsection{Working area and MLS description}

In order to test the described method, a LiDAR survey was carried out along an approximate length of 12 kilometres along two lanes of a main road. The objective of the survey is to use the clouds as the basis for cartography at 1:500 map scale, in order to support the road rehabilitation project. In addition to the two lanes of the main road, the crossing streets were also collected, so that the vector mapping cover up to 15 meters along each crossing street (Figure 6). 


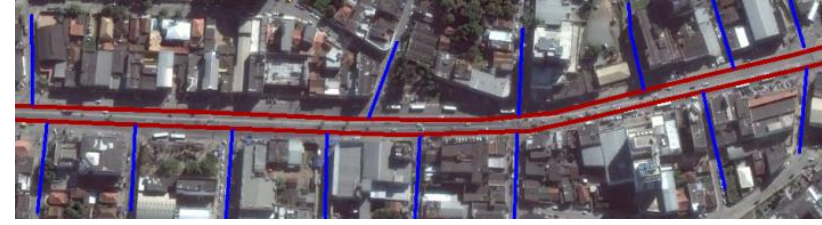

Figure 6. Main road in red and crossing streets in blue.

The MLS used was the Lynx M1 system from Optech. The system has a scan frequency of $500 \mathrm{KHz}$ and a mirror rotation speed of $200 \mathrm{~Hz}$. Inertial navigation was ensured by the Applanix Pos LV 520 system.

If in the main road it is expected some obstructions to the GNSS signal, the crossings streets are much narrower and flanked by tall buildings and so a much higher signal degradation is likely to produce very significant errors in the trajectory. Given the high number of crossing streets (106 identified along the $12 \mathrm{~km}$ of the main road) and the fact that it is not possible to use GNSS receivers due to the existing obstructions, the use of control points that allow the adjustment of the trajectory in each of the crossing streets can be quite time consuming and expensive (Gao et al, 2015). The proposed method will provide an alternative solution to this problem.

\subsection{Data collection and processing}

Both data collection and processing were performed in two separated blocks. The block A includes the two main road lanes and the block B include all crossings streets (Figure 7).

In each of the crossing streets, the data were collected in a way to guarantee an overlapping area with the clouds collected on the main road.

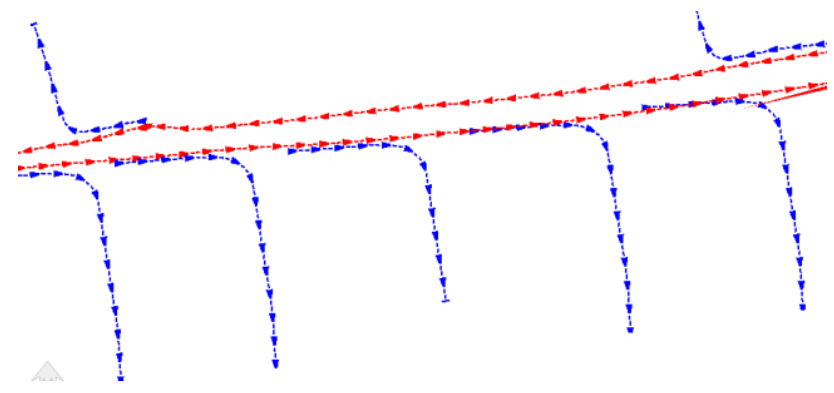

Figure 7. MLS vehicle trajectory (block A - red; block B blue).

For each block, the data was processed in two distinct phases according to the data type. First, the trajectory (POS files) was processed in order to obtain the trajectory positional correction, then this information was integrated with the information gathered by the LiDAR sensor to obtain the georeferenced point clouds. The trajectory processing was performed using the Applanix POSPAC Mobile Mapping Suite (POSPAC MMS), allowing the creation of the Smoothed Best Estimate of Trajectory (SBET) file. The Laser data processing was performed with Optech LiDAR Mapping Suite (LMS) software, and the result point clouds were stored in the standard format LAS 1.2.

\subsection{Point cloud registration}

It was intended to use the block A clouds as anchor and make the registration of the block $\mathrm{B}$ using the proposed method. The final use of the point clouds can determine the need of the absolute coordinates' registration of the anchor cloud. If the purpose of the clouds is to be used for cadastre of street signals, poles or other infrastructures, where there are no need for rigorous coordinates, it is possible to skip the absolute registration of the anchor cloud. In both cases the need of relative registration between the two blocks is required, to allow the application of automatic algorithms of object extraction or digital terrain models.

In this case, since the purpose of the clouds is to produce vector mapping at 1:500 map scale, absolute registration of block A was performed.

A set of 25 control points where used to perform the absolute registration of block A clouds. Once more, the use of this control points is not required for the proposed method. They were just used to unsure the absolute accuracy of the anchor clouds. If that accuracy can be assured for the trajectory values during the collecting process, or if the final purpose of the clouds does not require high accuracy, this step can be skipped.

The block A registration method is briefly described below, however the proposed method is only applied in the registration of block B.

The 25 control points where collected using dual frequency GNSS receivers (Figure 8) by post-processing method, with a minimum period of 20 minutes of observation. The points were always collected near the main road axis, where the obstructions to the GNSS signal are smaller.

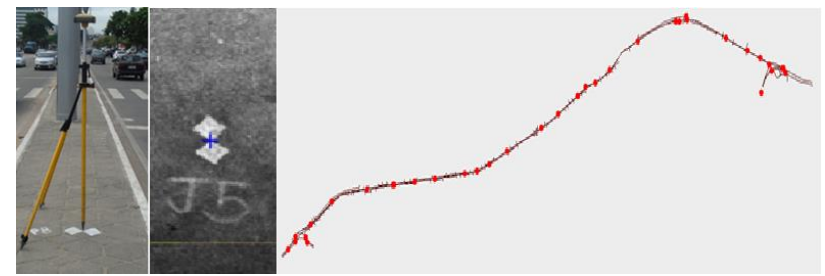

Figure 8. Control points along main road, in red.

The absolute registration of the point clouds of block A was made by adjustment of the trajectories, adding the information of the control points to the trajectory processing.

The POSPAC MMS was used to reprocessing the trajectory. The introduction of the control points as fixed positions to be integrated with other collected data allow the adjustment of the trajectory. To perform this process, POSPAC MMS needs to be fed with a file containing the coordinates of the sensor and the GPS time when the vehicle passes by the control point location. Using a point cloud viewer, each of the control points was identified in the cloud and its coordinates were measured. A C\# algorithm was implemented in order to get the GPS epoch for each control point, at the measured coordinates. Another module has been implemented to read the SBET file and return the coordinates of the trajectory at each epoch previously determined. Finally, the correction of the trajectory coordinates is applied, based on the difference between the absolute coordinates of each control point and the coordinates measured on the clouds in each passage of the trajectory. As a result of this process, a file with the fixed positions of the sensor along the trajectory is created. The file is used in the POSPAC MMS and the calculation of the new adjusted trajectory is obtained.

Before and after the adjustment, each control point (painted in the road) was identified and manually measured in the adjusted clouds.

In Table 1 the differences computed between the point cloud (manually measured) and the GNSS coordinates are presented for each control point, before and after the adjustment. 


\begin{tabular}{|c|c|c|c|c|c|c|}
\hline \multirow{2}{*}{$\begin{array}{c}\text { Control } \\
\text { point }\end{array}$} & \multicolumn{3}{|c|}{ Before adjustment } & \multicolumn{3}{|c|}{ After adjustment } \\
\hline & $\begin{array}{c}\text { Dif. } \\
\text { X }\end{array}$ & $\begin{array}{c}\text { Dif. } \\
\text { Y }\end{array}$ & $\begin{array}{c}\text { Dif. } \\
\text { Z }\end{array}$ & $\begin{array}{l}\text { Dif. } \\
X\end{array}$ & $\begin{array}{c}\text { Dif. } \\
\text { Y }\end{array}$ & $\begin{array}{c}\text { Dif. } \\
\text { Z }\end{array}$ \\
\hline 1 & 0.077 & 0.039 & 0.082 & 0.027 & 0.005 & 0.029 \\
\hline 2 & 040 & 0.071 & 0.098 & 0.008 & 0.013 & 0.040 \\
\hline 3 & 0.034 & 0.071 & 0.075 & 0.009 & 0.022 & 0.017 \\
\hline 4 & 0.096 & 0.126 & 0.188 & 0.018 & 0.024 & 0.022 \\
\hline 5 & 0.114 & 0.122 & 0.199 & 0.005 & 0.041 & 0.047 \\
\hline 6 & 0.117 & 0.098 & 0.162 & 0.028 & 0.012 & 0.032 \\
\hline 7 & 124 & 0.134 & 0.132 & 0.049 & 0.023 & 0.036 \\
\hline 8 & 0.092 & 0.057 & 0.086 & 0.015 & 0.027 & 0.024 \\
\hline 9 & 0.088 & 0.101 & 0.077 & 0.039 & 0.005 & 0.003 \\
\hline 10 & 0.074 & 0.027 & 0.023 & 0.026 & 0.000 & 0.004 \\
\hline 11 & 0.076 & 0.031 & 0.071 & 0.003 & 0.009 & 0.023 \\
\hline 12 & 035 & 0.030 & 0.062 & 0.001 & 0.007 & 0.022 \\
\hline 13 & 0.055 & 0.056 & 0.069 & 0.009 & 0.026 & 0.013 \\
\hline 14 & 0.004 & 0.069 & 0.070 & 0.001 & 0.012 & 0.026 \\
\hline 15 & 052 & 0.098 & 0.091 & 0.025 & 0.015 & 0.037 \\
\hline 16 & 0.059 & 0.064 & 0.089 & 0.025 & 0.016 & 0.007 \\
\hline 17 & 0.058 & 0.086 & 0.025 & 0.027 & 0.004 & 0.008 \\
\hline 18 & 0.030 & 0.044 & 0.099 & 0.012 & 0.018 & 0.003 \\
\hline 19 & 0.046 & 0.052 & 0.066 & 0.003 & 0.001 & 0.011 \\
\hline 20 & 0.073 & 0.015 & 0.081 & 0.030 & 0.003 & 0.019 \\
\hline 21 & 0.019 & 0.043 & 0.069 & 0.004 & 0.016 & 0.017 \\
\hline 22 & 0.061 & 0.023 & 0.073 & 0.012 & 0.008 & 0.005 \\
\hline 23 & 0.030 & 0.047 & 0.063 & 0.001 & 0.012 & 0.008 \\
\hline 24 & 0.068 & 0.034 & 0.085 & 0.014 & 0.000 & 0.010 \\
\hline 25 & 0.087 & 0.066 & 0.095 & 0.016 & 0.036 & 0.044 \\
\hline
\end{tabular}

Table 1. Differences computed between the manually measured point cloud and GNSS coordinates of each control point, before and after adjustment

Based in Table 1 values, it can be verified that the trajectory between control points 4 and 7 has the worse GNSS signal reception. After adjustment, the differences in all components are under $5 \mathrm{~cm}$.

After the adjustment, a new set of point clouds have been created, which represents the absolute registration of block A.

Hence, the registration of the block $\mathrm{B}$ was made using the proposed method, implemented through $\mathrm{C \#}$ algorithms.

For each cloud of block B, points that represent the trajectory of the vehicle (Equation 1) were defined, a time interval $d=0.25$ seconds has been set, as well as the minimum distance between consecutive points $(\Delta)$ with 2 meters. A value of 15 centimetres was used as the radius $r$. This circle was applied in both clouds of the block A and of the block B in order to identify the points that allowed the definition of the planes (RANSAC) in each one of the clouds. The $r$ value can be adjusted based on the density of the cloud, and different values of $r$ can be used for anchor and adjusted cloud, respectively. Based on the RANSAC probability value, it is possible to reject some of the points and/or adapt its values.
The definition of all planes allow the establishment of the differences between the clouds in each of the trajectory point. Figure 9 shows an example of the overlapping area of a main road cloud (purple) and a cloud of a crossing street (yellow).

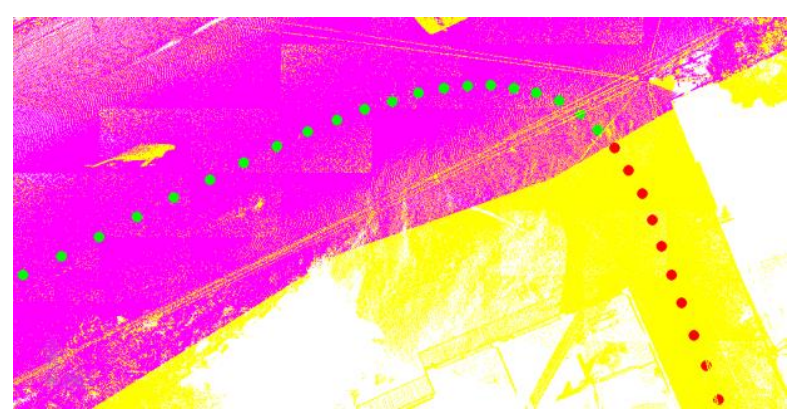

Figure 9. Overlapping area of main road cloud (purple) and a crossing street (yellow).

Along the crossing street cloud there is no overlap with the main cloud and consequently it is not possible to apply the method (red dots, Figure 9). In the remaining points of the trajectory of the crossing street cloud, outside the overlapping limit, we decide to apply the correction corresponding to the last overlapping point (Figure 10).

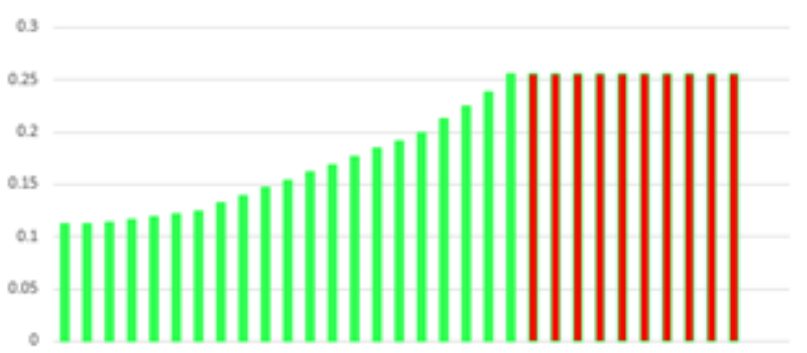

Figure 10. Values in meters to be applied for each of the points along the trajectory

After the registration of all clouds of block B, a maximum vertical difference of $2 \mathrm{~cm}$ was measured along the trajectory. This value is detected between the clouds of block B relative to the clouds of block A. The differences measured before the registration varied between 5 and $55 \mathrm{~cm}$. A longitudinal profile along a crossing street trajectory before and after the registration is presented in Figure 11.

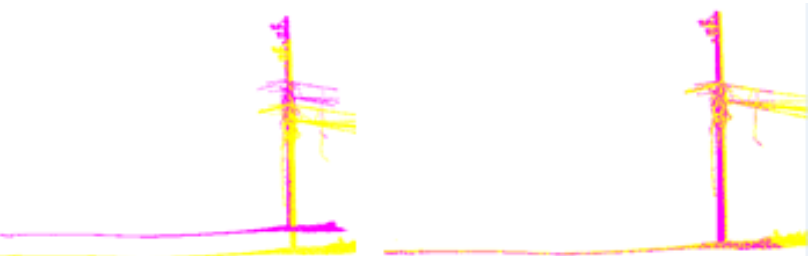

Figure 11. Vertical differences before and after the registration process.

However, differences were detected in some adjusted clouds, when compared with areas further away from the adjusted trajectory. These differences may result from IMU errors or from initial boresight misalignments. In Figure 12, a cross section is shown in the overlapping area between the two clouds, before 
and after the registration process. It can be verified that there is an inclination angle of the block B cloud relative to the block A cloud, symmetrically centred in the trajectory point.

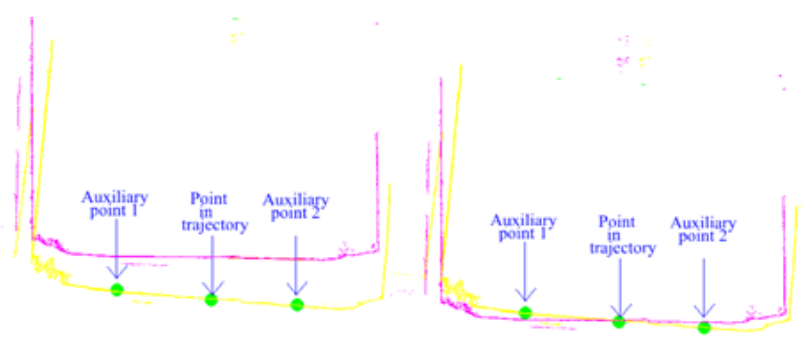

Figure 12. Cross section showing the perpendicular angle of the point cloud, after registration.

To eliminate these differences, a variant of the proposed method was introduced. In addition to the correction of the vertical component, two new points along the sensor reading profile were inserted (Figure 12). These two points were easily added by changing the angle $\alpha$ (Equation 1). In this case, the registration was performed along the trajectory not only with a point but with 3 points (Figure 13).

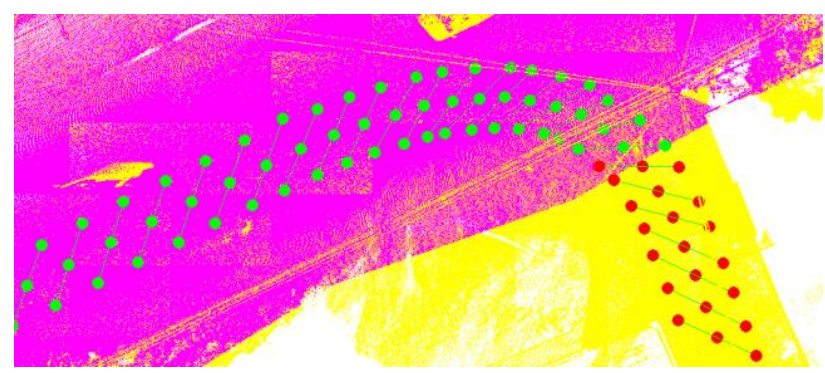

Figure 13. Point's triplet along trajectory

The points are not perpendicular to the trajectory, since the sensor is placed at a 45 degree angle to the vehicle trajectory, and the points are in the scanning direction of the sensor. Since the coordinates of the auxiliary points are known, it is possible to calculate the distance between the trajectory point and each of the auxiliary points. Using the differences between the two blocks clouds at each point, it is possible to calculate the transverse angle (Equation 3). Between two epochs used in the trajectory, the inclination angle $(\rho)$ value was linearly interpolated in the same way as the vertical difference (Equation 2). It was found that the angle calculated using any of the auxiliary points was very similar, confirming that the cloud slope error should result from a sensor boresight misalignment.

$\rho=\cos \left(\frac{\text { Dif }_{a 1}-D i f_{p}}{\text { Dist }_{a 1}}\right) \cong \cos \left(\frac{\text { Dif }_{a 2}-D i f_{p}}{\text { Dist }_{a 2}}\right)$

where $\quad \rho=$ inclination angle

Dif $_{\mathrm{p}}=$ difference between clouds at trajectory point

Dif $_{\mathrm{a} 1}$, Dif $_{\mathrm{a} 2}=$ differences between clouds at auxiliary points 1 and 2

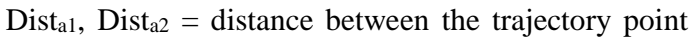
and auxiliary points 1 and 2 .

After applying the variant to the method, including the perpendicular angle correction, new measurements show that the differences parallel to the trajectory significantly decreased. Still they are bigger than the differences along trajectory, reaching 4 centimetres and they proportionally increase with the distance to the trajectory.

\subsection{Obtained results}

A time interval of $0.3 \mathrm{~s}$ was used to establish the points for the adjustment of the 106 crossing streets clouds. This procedure resulted in the creation of 5406 points (including the points along the trajectory and the two corresponding correction angle points). Planes were created in the crossing streets clouds and main road clouds, both centred in each point. After finishing the adjustment, the planes were created again, at the same points, now using the new LAS files containing the adjusted clouds.

Table 2 shows the statistics of absolute planes difference before and after the adjustment.

\begin{tabular}{|c|c|c|c|c|c|}
\cline { 2 - 6 } \multicolumn{1}{c|}{} & $\begin{array}{c}\text { Min. } \\
\text { Value }\end{array}$ & $\begin{array}{c}\text { Max. } \\
\text { Value }\end{array}$ & $\begin{array}{c}\text { Min. } \\
\text { cross } \\
\text { road } \\
\text { average }\end{array}$ & $\begin{array}{c}\text { Max. } \\
\text { cross } \\
\text { road } \\
\text { average }\end{array}$ & $\begin{array}{c}\text { All } \\
\text { points } \\
\text { average }\end{array}$ \\
\hline $\begin{array}{c}\text { Before } \\
\text { adjust. }\end{array}$ & $0.05 \mathrm{~m}$ & $0.55 \mathrm{~m}$ & $0.09 \mathrm{~m}$ & $0.45 \mathrm{~m}$ & $0.29 \mathrm{~m}$ \\
\hline $\begin{array}{c}\text { After } \\
\text { adjust. }\end{array}$ & $0.001 \mathrm{~m}$ & $0.02 \mathrm{~m}$ & $0.005 \mathrm{~m}$ & $0.015 \mathrm{~m}$ & $0.01 \mathrm{~m}$ \\
\hline
\end{tabular}

Table 2. Statistics of automatic differences between the created planes, before and after the adjustment

It should be noted that the differences presented in Table 2 refer to the planes centred exactly at the points used in the adjustment. In order to perform an independent check of the points used in the adjustment, three manual points were measured in each of the cross roads adjusted cloud. The points were established at approximately the clouds' overlapping boundary. One of the points was measured approximately along the trajectory and the other two, one of each side, transversely to the trajectory (Figure 14), in a total of 318 locations.

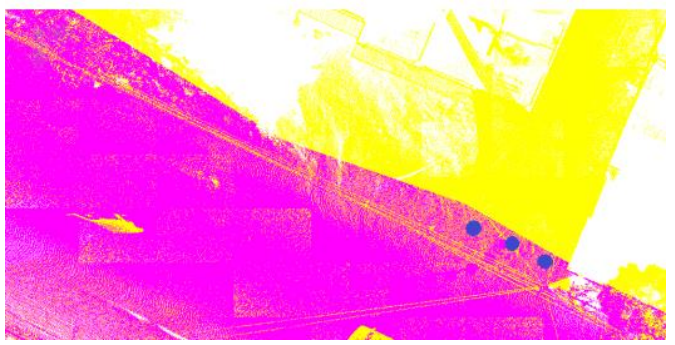

Figure 14. Approximate location of manual control point triplet

At each of these locations, the elevation of the nearest point in each cloud (main road and crossing Street) was manually measured. Table 3 shows the statistics related to the differences found.

\begin{tabular}{|c|c|c|c|c|c|}
\hline $\begin{array}{c}\text { Points } \\
\text { number }\end{array}$ & $\begin{array}{c}\text { Min. } \\
\text { Value }\end{array}$ & $\begin{array}{c}\text { Max. } \\
\text { Value }\end{array}$ & $\begin{array}{c}\text { Min. } \\
\text { cross } \\
\text { road } \\
\text { average }\end{array}$ & $\begin{array}{c}\text { Max. } \\
\text { cross } \\
\text { road } \\
\text { average }\end{array}$ & $\begin{array}{c}\text { All } \\
\text { points } \\
\text { average }\end{array}$ \\
\hline 318 & $0.01 \mathrm{~m}$ & $0.12 \mathrm{~m}$ & $0.11 \mathrm{~m}$ & $0.02 \mathrm{~m}$ & $0.045 \mathrm{~m}$ \\
\hline
\end{tabular}

Table 3. Statistics of the manual measured differences between the main road clouds and the adjusted crossing roads clouds 
The greatest differences were found in points far away from the cloud trajectories. In points close to the trajectory, the maximum difference found was $4 \mathrm{~cm}$.

\section{CONCLUSIONS AND FUTURE WORK}

A new method is proposed to make registration of clouds collected from a MLS. The method has proved to work with good results, making relative registration of point clouds. The process almost eliminates the differences of a cloud relative to another anchor cloud, using the overlapping area. Based in the reconstruction and adjustment of the trajectory, the process is able to eliminate the vertical differences between the clouds in a fully automatic process.

A variant process to correct the perpendicular inclination of the clouds was successfully applied.

The process reveal to be very fast, allowing the registration of a 50 million point cloud in less than one minute. The flexibility of the process allows the user to perform the registration in one or several point clouds.

Additionally, it was demonstrated that the information contained in the LAS standard file is now suitable for make the registration process. Hopefully, the dissemination and implementation of this kind of methods will increase the standardization of the point cloud file format. Nowadays, many companies and researchers are just specialized in extracting information from the point clouds. The fact that the process uses solely information contained in the LAS standard files represents an advantage for the cases that does not need any additional information besides the LAS files to perform some clouds registration. During the research of this proposed method, different implementation of LAS from different MLS producers were tested. It was verified that many fields of the format are empty or do not respect the LAS standard rules. In future work a review of those implementation standards will be an added value.

Future work that includes the test of the proposed method applied to the horizontal registration of the clouds is recommended. This should be a successful task, due to the simplicity of the process. In future work, the behaviour of the trajectory outside the overlapping area after the registration process should also be investigated. Since a Kalman filter is applied to the trajectory, it is expected that outside the overlapping area the cloud is still usable for some extra meters, if a suitable interpolation function is applied.

\section{REFERENCES}

Abuhadrous, I., Nashashibi, F., Goulette, F., Laurgeau, C., 2003. 3-D Land Vehicle Localization: a Real-time MultiSensor Data Fusion Approach using RTMAPS. In Proceedings of the 11th International Conference on Advanced Robotics. June 30th July 3rd, University of Coimbra, Portugal.

ASPRS, 2013. ASPRS LAS 1.4 Format Specification, http://www.asprs.org/wpcontent/uploads/2010/12/LAS_1_4_r1 3.pdf (accessed 20 January 2017)

El-Sheimy, N., 2005. An overview of mobile mapping systems, From Pharaos to Geoinformatics. FIG Working Week 2005 and GSDI-8, Cairo, Egypt, April 16-21.

Fischler, M. A., Bolles, R.C., 1981. Random sample consensus: A paradigm for model fitting with applications to image analysis and automated cartography. Communications of the ACM, Vol. 24, pp. 381-395.
Gao, Y., Huang, X., Zhang, F., Fu, Z., Yang, C., 2015. Automatic Geo-referencing Mobile Laser Scanning Data to UAV images. The International Archives of the Photogrammetry, Remote Sensing and Spatial Information Sciences, Toronto, Canada, Vol. XL-1/W4, pp. 41-46.

González-Jorge, H., Martínez Sánchez, J., Díaz-Vilariño, L., Puente, I., Arias, P. 2016. Automatic Registration of Mobile LiDAR Data Using High-Reflectivity Traffic Signs. Journal of Construction Engineering and Management, 04016022. DOI: 10.1061/(ASCE)CO.1943-7862.0001143.

Gressin, A., Cannelle, B., Mallet, C., Papelard J., 2012. Trajectory-based registration of 3D LiDAR point clouds acquired with a mobile mapping system. ISPRS Annals of the Photogrammetry, Remote Sensing and Spatial Information Sciences, Melbourne, Australia, Vol. I-3, pp. 117-122.

Gressin, A., Mallet, C., Demantk, J., David, N., 2013. Towards 3D LiDAR point cloud registration improvement using optimal neighborhood knowledge. ISPRS Journal of Photogrammetry and Remote Sensing, 79, pp. 240-251.

Pomerleau, F., Colas, F., Siegwart, R., 2015. A Review of Point Cloud Registration Algorithms for Mobile Robotics. Foundations and Trends in Robotics, Now Publishers, 4(1), pp.1104.

Puente, I., Solla, M., González-Jorge, H., Arias, P., 2013. Validation of mobile LiDAR surveying for measuring pavement layer thicknesses and volumes. NDT \& E International, Vol. 60, pp. $70-76$.

Samberg, A., 2007. An implementation of the ASPRS LAS standard. IASPRS, Vol. XXXVI, Part 3 / W52, pp. 363-371.

Schaer, P., Vallet, J., 2016. Trajectory adjustment of mobile laser scan data in GPS denied environments. The International Archives of the Photogrammetry, Remote Sensing and Spatial Information Sciences, Lausanne, Switzerland, Vol. XL-3/W4, pp. 61-64.

Wang, R., Ferrie, F., 2015. Automatic registration method for mobile LiDAR data, Optical Engineering, 54(1), 013108. DOI: 10.1117/1.OE.54.1.013108. 